Vol. 11, No. 40, July, 2016, 1084-1090

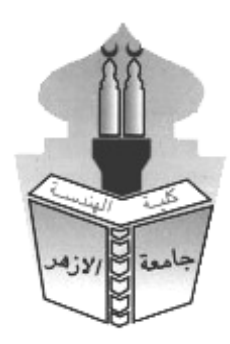

\title{
BLINDNESS ELECTRONIC ASSISTANT SYSTEM
}

\author{
Ayman A. Nassar ${ }^{1}$, and Yasser M. Kadah ${ }^{2}$ \\ ${ }^{1}$ Military Technical College and Cairo University ${ }^{2}$
}

\begin{abstract}
Electronic assistant system for blind people is introduced which is capable of detecting obstacles around the blind person allowing him to walk, deal and react with the surrounding environment independently. Ultrasound and IR distance sensors are used in this development to detect the distance of obstacles surrounding the blind person according to the sensors distribution. The system here will convert the sensor signal to vocal signal and vibration to be accessible for blind person hearing nearby obstacles as high frequency beep and further obstacles as low frequency beep, Vibration motors will be set in the two sides of the blind person indication for left or right obstacles. This study shows a design and implementation of assistant system which will be helpful for blind persons who always suffer from being dependent on someone for help or doing their stuff.
\end{abstract}

\section{KEYWORDS: Blindness, Assistant system, Ultrasound distance sensor, Obstacles, Servo motor.}

I. INTRODUCTION

Blindness problem is one of the most challenges faced by any country. Every 5 seconds, one person goes blind in the world which leads to 39 million blind and 246 million people have low vision about $90 \%$ of the visually impaired live in developing countries [1]. Blind persons seek for feeling independent as they want to walk on streets, cross the road, and getting their special stuff without waiting help from others especially if someone changes their location, also indoor navigation and orientation difficulties are: missing known landmarks, restrictions to use guide dogs, overcoming obstacles can be risky [2]. Normal person interact and learn from all five senses as sight makes up $83 \%$ of the impact on the brain of information from the senses during a visual scene, Taste makes up 1\%, Hearing makes up 11\%, Smell makes up 3\%, and touch 2\% [3]. Human body is an open bio-system, it acts and reacts with the environment, exchange different types of energies. Sense and communicate with others. Development of a system that is capable of converting the impairment or disability vision to other available compensatory sense is very important for enabling the blind person feel familiar with the surrounding environment independently without facing any obstacles hurting him.

Related works study a number of indoor and outdoor navigation and way finding techniques for visually impaired persons. [4]Whereas outdoor systems rely upon GPS to locate the user's location $[5,6]$, indoor systems typically rely upon physical augmentation of the environment such as infrared (IR) [7, 8], ultrasound [9], or radio frequency identifier (RFID) tags [10, 11, 12] or expensive sensing equipment such as the systems based on computer vision [13]. 


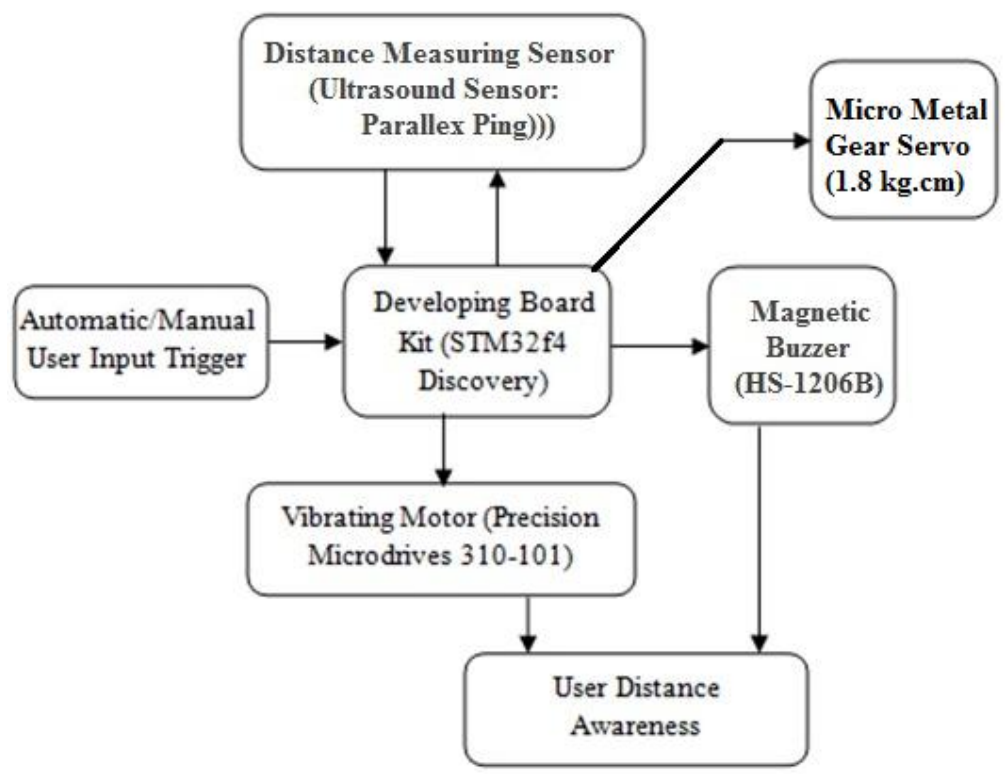

Fig. 1. Block diagram shows the main items of the Blind Electronic Assistant portable devices and the relationship between each others.

Fig.1 Shows that the paper presenting a portable device that contains an ultrasound distance sensor which sends ultrasound signal and receive its echo, Developing tool kit is used for signal processing, conversion and then activate a vocal signal and vibration motor to be accessible to blind people indicating the distance and the direction of obstacles as a compensatory way instead of vision. Sensor will send its signals in three directions (in front and at the two sides) to make the blind person aware of obstacles around him.

\section{MATERIALS AND METHODS}

\section{A. Ultrasound Distance Sensor}

The ultrasound sensor consists of two parts. An emitter which produces a $40 \mathrm{kHz}$ wave and a detector detects the echo of the emitted wave.

Fig. 2 shows the operational time line of the ping ultrasound sensor by sending the ultrasound burst wave that travel through air at about 1130 feet per second then reflected back when facing any obstacles bounce back to the sensor after a trip delay [14].

After sending the emitted wave, the ping ultrasound sensor provides an output pulse to the host that will end when the echo is detected so the period or width of this pulse is the trip delay corresponds to the distance to the target.

\section{B. Magnetic Buzzer}

HS1206B magnetic buzzer is used and drive by electrical signal with specified frequency according to the tone needed corresponding to the distance measured by the ultrasound sensor. 

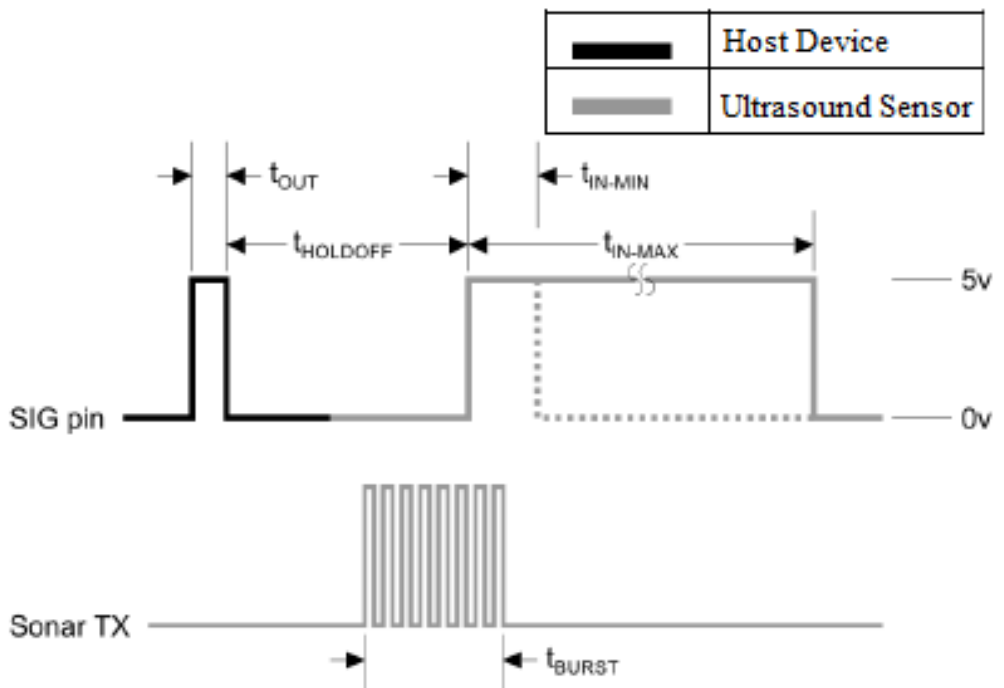

Fig. 2. The Operation Time Line Of The Ultrasound Sensor, The Variables: $T_{\text {out }}$, Input Trigger Pulse $5 \mu$; $T_{\text {holdoff }}$ Echo Holdoff $750 \mu$ s; $T_{\text {burst }}$, Burst Frequency $200 \mu$ s At 40khz; $T_{\text {in-MIN }}$, Echo Return Pulse Minimum

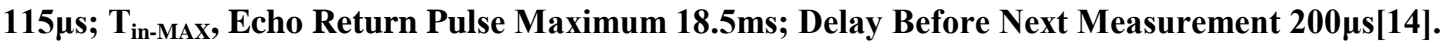

\section{Vibration Motor}

Vibration motor model no. (310-101) is used in the system and it is a shaft-less vibratory motor which is perfect for non-audible indicators and can be used in any number of applications[15], in this study it is used as a vibrating indicator perfect for blind deaf persons, the length of vibrating pulse is corresponding to the distance measured by the ultrasound sensor.

\section{Micro Metal Gear Servo}

It is a micro size metal gear servo motor $(1.8 \mathrm{Kg} . \mathrm{cm})$, it is unlike dc motors, as we can position the motor shaft at a specific position (angle) using control signal. The motor shaft will hold at this position as long as the control signal not changed. This is very useful in our system as by fixing the ultrasound sensor on the servo motor, and rotates its shaft in different angles that allows the sensor to send its ultrasound signal in different angles to detect obstacles around the blind person.

\section{E. STM32F4 Discovery Developing Kit}

STM32F4 Discovery is high performance developing kit, it contains 32-bit ARM Cortex-M4f core, MEMS motion sensor, MEMS audio sensor, audio DAC speaker driver, 8 LEDs, Two push buttons, and micro-AB USB connector [16].

This board is very helpful in development any application easily, as it includes almost everything required for beginners and experienced users to get started quickly [16]. This board has many development tools, but in this design Embedded Workbench for ARM (EWARM) is used.

\section{EXPERIMENTAL PROCEDURES}

A. Ultrasound Assistant system experiment

The experiment was done by connecting an ultrasound ping sensor to the developing board kit to send the ultrasound signal and receive back the echo signal, headphone speaker and two vibration motor are also connected to the developing board kit to be as compensatory ways to be available by the blind persons. 


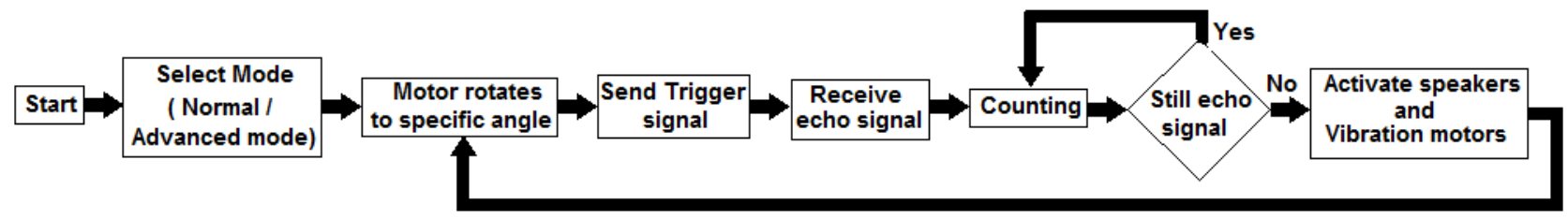

Fig. 3. The flow chart block diagram shows that the program begins by pressing on the start button which trigger the ultrasound sensor then receive the echo signal and the time delay is the trip time, counting this time is used to control the frequency of beep done by the speakers and to control the vibration motors.

Fig.3 Shows the Flow chart block diagram which start by pressing the start button, the developing board kit was programmed by our software design using EWARM to control the whole circuit, After pressing the start button, the ultrasound signal sends a burst of ultrasound wave which will travel through air until it faces an obstacles and this burst reflected back to the sensor, the sensor gives high output until this echo signal returns back so this period of time is the trip time so all the distance taken by the signal is assigned as

$$
\text { All Distance }=\text { Velocity } * \text { Time }
$$

As the trip time is the time from the sensor to the obstacle and return back to the sensor so equation (1) will be assigned as

$$
\text { Distance }=\frac{\text { Velocity } * \text { Time }}{2}
$$

fter calculating the distance from (2) then the system will activate the speakers and also activate the vibrating motors with a beeping frequency or vibrating frequency corresponding to the distance measured.

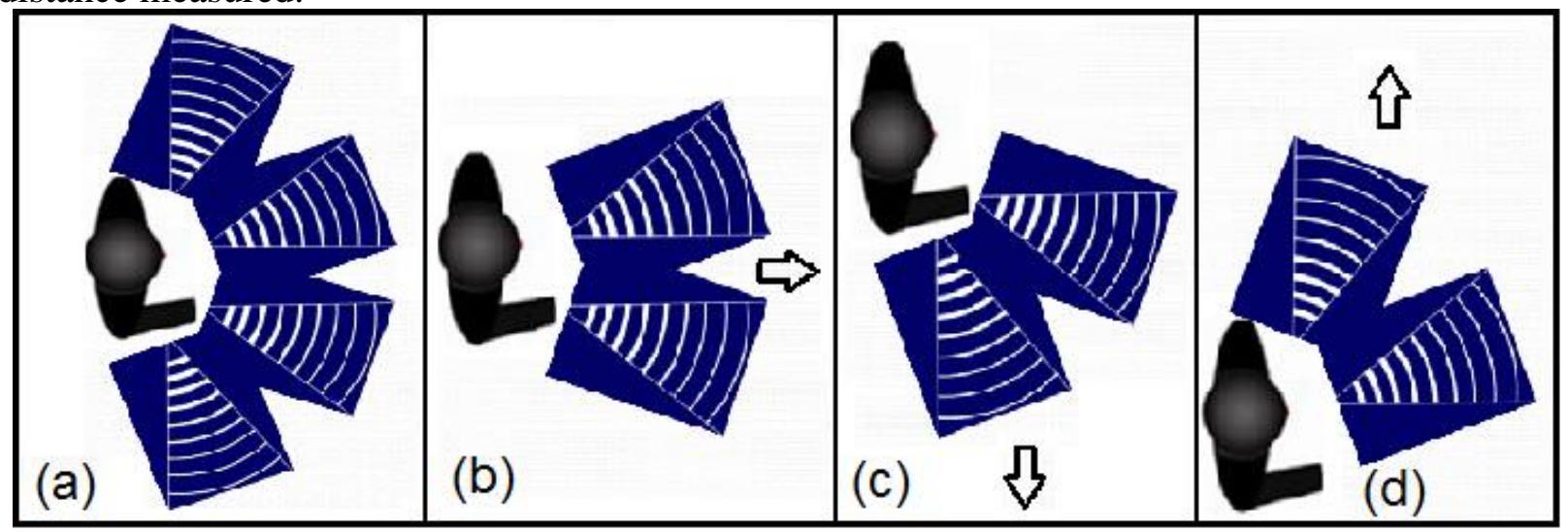

Fig.4. (a) This figure shows normal navigation mode which sends ultrasound waves in all 1800 regardless the any direction of motion, figure (b), (c),(d) shows the advanced navigation mode which sends only ultrasound waves in 900 and according to the direction of the blind person motion.

\section{B. Navigation mode selection}

The main aim of the experiment is to cover the all area $\left(180^{\circ}\right)$ around the blind person, In other studies they used multiple sensors in all directions of interest to be sure that no obstacles will be missed from ultrasound scanning, In this system only one ultrasound distance sensor is used but it is fixed on small size servo motor that rotates in fixed angles to detect obstacles around the blind person.

The system is enhanced by adding a Navigation mode selection option that minimizes the scanned area by the ultrasound distance sensor from $180^{\circ}$ to $90^{\circ}$ in the direction of the motion of the blind person by the blind persons. 
Fig.4(a) Shows how the normal mode works as shown in the picture that the ultrasound distance sensor sends its waves in at angle of $180^{\circ}$ around the blind person, Fig.4(b),(c),(d) shows how the advanced navigation mode works as it depends on the motion sensors inside the developing kit (STM32F4 Discovery) to detect the direction of motion of the blind person, From this information the program automatically set the angles which the servo motor will take consequentially the ultrasound distance sensor will send its waves only in the angle of motion and neglect the less important angles.

\section{Obstacles}

The selection of the obstacles was done according to variety of size and shape (Rectangular and Cylindrical shapes), and all the obstacles are rigid, the sensor and obstacles are on 1meter height from the ground to avoid reflections from the floor.

Fig.5 shows the typical picture of the obstacles including the wall as an obstacle too, the obstacles were put on a carefully chosen table with a thin area towards the sensor for not being an obstacles itself. $(1.5 \mathrm{~cm} * 80 \mathrm{~cm})$.

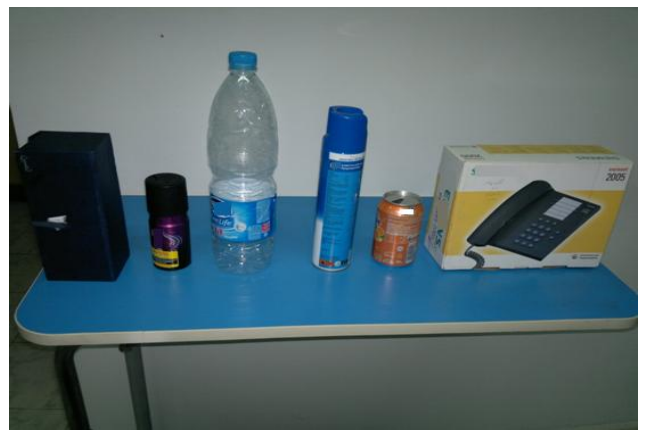

Fig.5. The typical picture of the obstacles which were chosen according to different shape and size and all are

3. EXPERIMENTAL RESULTS rigid.

The experiment was done on every obstacle alone but in the two ways. First experiment was done by fixing the angle of the sensor and obstacle such that the sensor faces the obstacles and change the distance between the sensor and obstacles.

\begin{tabular}{|c|c|c|c|c|c|c|c|c|c|c|}
\hline & \multicolumn{6}{|c|}{ Flat Surfaces } & \multicolumn{4}{|c|}{ Cylindrical surfaces } \\
\hline & Wall & $24 * 18$ & $18 * 24$ & $8 * 24$ & $8 * 20$ & $8 * 7$ & L32*D8 & L25*D6 & $\mathrm{L} 14 * \mathrm{D} 5$ & $\mathrm{~L} 11 * \mathrm{D} 7$ \\
\hline $3 \mathrm{~cm}$ & $\sqrt{ }$ & $\sqrt{ }$ & $\sqrt{ }$ & $\sqrt{ }$ & $\sqrt{ }$ & $\sqrt{ }$ & $\sqrt{ }$ & $\sqrt{ }$ & $\sqrt{ }$ & $V$ \\
\hline $6 \mathrm{~cm}$ & $\sqrt{ }$ & $\sqrt{ }$ & $\sqrt{ }$ & $\sqrt{ }$ & $\sqrt{ }$ & $\sqrt{ }$ & $\sqrt{ }$ & $\sqrt{ }$ & $\sqrt{ }$ & $\sqrt{ }$ \\
\hline $10 \mathrm{~cm}$ & $\sqrt{ }$ & $\sqrt{ }$ & $\sqrt{ }$ & $\sqrt{ }$ & $\sqrt{ }$ & $\sqrt{ }$ & $\sqrt{ }$ & $\sqrt{ }$ & $\sqrt{ }$ & $\sqrt{ }$ \\
\hline $15 \mathrm{~cm}$ & $\sqrt{ }$ & $\sqrt{ }$ & $\sqrt{ }$ & $\sqrt{ }$ & $\sqrt{ }$ & $\sqrt{ }$ & $\sqrt{ }$ & $\sqrt{ }$ & $\sqrt{ }$ & $\sqrt{ }$ \\
\hline $20 \mathrm{~cm}$ & $\sqrt{ }$ & $\sqrt{ }$ & $\sqrt{ }$ & $\sqrt{ }$ & $\sqrt{ }$ & $\sqrt{ }$ & $\sqrt{ }$ & $\sqrt{ }$ & $\sqrt{ }$ & $\sqrt{ }$ \\
\hline $30 \mathrm{~cm}$ & $\sqrt{ }$ & $\sqrt{ }$ & $\sqrt{ }$ & $\sqrt{ }$ & $\sqrt{ }$ & $\sqrt{ }$ & $\sqrt{ }$ & $\sqrt{ }$ & $\sqrt{ }$ & $\sqrt{ }$ \\
\hline $50 \mathrm{~cm}$ & $\sqrt{ }$ & $\sqrt{ }$ & $\sqrt{ }$ & $\sqrt{ }$ & $\sqrt{ }$ & $\sqrt{ }$ & $\sqrt{ }$ & $\sqrt{ }$ & $\sqrt{ }$ & $\sqrt{ }$ \\
\hline $70 \mathrm{~cm}$ & $\sqrt{ }$ & $\sqrt{ }$ & $\sqrt{ }$ & $\sqrt{ }$ & $\sqrt{ }$ & $\sqrt{ }$ & $\sqrt{ }$ & $\sqrt{ }$ & $\sqrt{ }$ & $\sqrt{ }$ \\
\hline $100 \mathrm{~cm}$ & $\sqrt{ }$ & $\sqrt{ }$ & $\sqrt{ }$ & $\sqrt{ }$ & $\sqrt{ }$ & $\sqrt{ }$ & $\sqrt{ }$ & $\sqrt{ }$ & $\sqrt{ }$ & $\sqrt{ }$ \\
\hline $130 \mathrm{~cm}$ & $\sqrt{ }$ & $\sqrt{ }$ & $\sqrt{ }$ & $\sqrt{ }$ & $\sqrt{ }$ & $\sqrt{ }$ & $\sqrt{ }$ & $\sqrt{ }$ & $\sqrt{ }$ & $\sqrt{ }$ \\
\hline $160 \mathrm{~cm}$ & $\sqrt{ }$ & $\sqrt{ }$ & $\sqrt{ }$ & $\sqrt{ }$ & $\sqrt{ }$ & $\sqrt{ }$ & $\sqrt{ }$ & $\sqrt{ }$ & $\sqrt{ }$ & $\sqrt{ }$ \\
\hline $200 \mathrm{~cm}$ & $\sqrt{ }$ & $\sqrt{ }$ & $\sqrt{ }$ & $\sqrt{ }$ & $\sqrt{ }$ & $\sqrt{ }$ & $\sqrt{ }$ & $\sqrt{ }$ & $\sqrt{ }$ & $\sqrt{ }$ \\
\hline $220 \mathrm{~cm}$ & $\sqrt{ }$ & $\sqrt{ }$ & $\sqrt{ }$ & $\sqrt{ }$ & $\sqrt{ }$ & $\sqrt{ }$ & $\sqrt{ }$ & $\cdots$ & - & - \\
\hline $250 \mathrm{~cm}$ & $\sqrt{ }$ & $\sqrt{ }$ & $\sqrt{ }$ & $\sqrt{ }$ & $\sqrt{ }$ & $\sqrt{ }$ & $\cdots$ & - & $\cdots$ & $\cdots$ \\
\hline $270 \mathrm{~cm}$ & $\sqrt{ }$ & $\sqrt{ }$ & $\sqrt{ }$ & $\cdots$ & $\cdots$ & $\cdots$ & $\cdots$ & $\cdots$ & -- & $\cdots$ \\
\hline $300 \mathrm{~cm}$ & $\sqrt{ }$ & $\sqrt{ }$ & $\sqrt{ }$ & $\cdots$ & $\cdots$ & $\cdots$ & $\cdots$ & $\cdots$ & $\cdots$ & $\cdots$ \\
\hline
\end{tabular}

Table1. The results of the obstacles of different size and shape that the sensor can detect in case of the sensor in front of the obstacle. 
Table 1 shows that the type and size of obstacles that can be detected when the sensor faces the obstacles in different distances, flat surfaces can be detected more than cylindrical surfaces in farther distances, The aim of this experiment is to see how the blindness electronic assistant system can detect even small objects of different sizes and shapes.

The system can detect obstacles even they were very small or their shape were flat or cylindrical at a distance of 2 meter or less, that gives the blind person a plenty of time when walking to stop and avoid the detected obstacle.

\begin{tabular}{|c|c|c|c|c|c|c|c|c|c|c|}
\hline & \multicolumn{5}{|c|}{ Flat Surfaces } & \multicolumn{4}{c|}{ Cylindrical surfaces } \\
\hline & Wall & $24 * 18$ & $18 * 24$ & $8 * 24$ & $8 * 20$ & $8 * 7$ & L32*D8 & L25*D6 & L14*D5 & L11*D7 \\
\hline $10^{\circ}$ & $\sqrt{ }$ & $\sqrt{ }$ & $\sqrt{ }$ & $\sqrt{ }$ & $\sqrt{ }$ & $\sqrt{ }$ & $\sqrt{ }$ & $\sqrt{ }$ & $\sqrt{ }$ & $\sqrt{ }$ \\
\hline $20^{\circ}$ & $\sqrt{ }$ & $\sqrt{ }$ & $\sqrt{ }$ & - & - & -- & $\sqrt{ }$ & $\sqrt{ }$ & $\sqrt{ }$ & $\sqrt{ }$ \\
\hline
\end{tabular}

Table2. The results of the obstacles of different size and shape that the sensor can detect in case of the distance between the sensor and obstacle is fixed (1 meter) but with different angles to the obstacle.

Second experiment was done by changing the angle between the sensor and obstacle but fixing the distance between them to 1meter, the angles were chosen as $10 \mathrm{o} \& 20 \mathrm{o}$ since more than that the sensor gives poor data.

The cylindrical obstacles give better detection than flat obstacles especially small ones but large flat obstacles can be detected.

\section{Conclusion}

The results presented in this paper shows that the Blindness Electronic Assistant System is capable of detecting obstacles for blind persons and also using only one sensor fixed on a servo motor gives high efficiency than using multiple sensors, as the experiments results show that the maximum suitable angle of detection is about $10^{\circ}$ in both sides which means that in case of using multiple sensors it needs to use 9 ultrasound sensors to cover $180^{\circ}$ otherwise undetectable zones will appear. Rotating sensor makes the system more accurate, cheaper, light in weight and less complex.

\section{REFERENCES}

[1] WHO Media Centre, "Visual impairment and Blindness," fact sheet, $N^{\circ} 282$, June. 2012.

[2] Rosen S. Ivanov, A LOW-COST INDOOR NAVIGATION SYSTEM FOR VISUALLY IMPAIRED AND BLIND, Communication \& Cognition, Vol. 44, Nr. 3.2011

[3] B. Pease, and A. Pease, The Definitive Book of Body Language, Pease International Press: Australia, 2004, pp. 189.

[4] N. Fallah, A Mixed Reality Navigation System for Individuals Who Are Visually Impaired, SIGACCESS NEWSLETTER, ISSUE 96, JAN 2010,pp.24-27

[5] Arikawa, M., Konomi, S., and Ohnishi, K. Navitime: Supporting pedestrian navigation in the real world. IEEE Pervasive Computing 6, 3 (2007), pp. 21-29.

[6] Strachan, S., Williamson, J., and Murray-Smith, R. Show me the way to monte carlo: densitybased trajectory navigation. In CHI '07: Proceedings of the SIGCHI conference on Human factors in computing systems (2007), ACM, pp. 1245-1248.

[7] Sonnenblick, Y, An indoor navigation system for blind individuals, In Proceedings of the 13th annual Conference on Technology and Persons with Disabilities .1998.

[8] Ross, D. A., and Lightman, A. Talking braille: a wireless ubiquitous computing network for orientation and wayfinding. In Assets '05: Proceedings of the 7th international ACM SIGACCESS conference on Computers and accessibility (2005), ACM, pp. 98-105.

[9] Ran, L., Helal, S., and Moore, S. Drishti: An integrated indoor/outdoor blind navigation system and service, Pervasive Computing and Communications, IEEE International Conference. 2004,pp. 23. 
[10] Amemiya, T., Yamashita, J., Hirota, K., and Hirose, M. Virtual leading blocks for the deafblind: A real-time way- nder by verbal-nonverbal hybrid interface and high-density red tag space. In VR '04: Proceedings of the IEEE Virtual Reality 2004 (2004), IEEE Computer Society, p. 165.

[11] Gifford, S., Knox, J., James, J., and Prakash, A. Introduction to the talking points project. In Assets '06: Proceedings of the 8th international ACM SIGACCESS conference on Computers and accessibility (2006), ACM, pp. 271-272.

[12] Bessho, M., Kobayashi, S., Koshizuka, N., and Sakamura, K. Assisting mobility of the disabled using space-identifying ubiquitous infrastructure. In Assets '08: Proceedings of the 10th international ACM SIGACCESS conference on Computers and accessibility (2008), ACM, pp. 283-284.

[13] Hub, A., Diepstraten, J., and Ertl, T. Design and development of an indoor navigation and object identifcation system for the blind. SIGACCESS Access. Comput., 77-78. 2004, pp. 147-152.

[14] Ping))) Ultrasonic distance sensor Datasheet,No.28015, v2.0, 2/4/2013, Parallax Inc.

[15] Vibration motor model no. 310-101 Datasheet2008, Precision Microdrives.

[16] STM32F4 high-performance discovery board Data brief, Doc ID $022204 \mathrm{Rev}$ 1, September 2011, ST Microelectronics. 